REF - ISSN 1808-0804 Vol. VI (1), 14 - 24, 2009

\title{
FÁRMACOS: DO DESENVOLVIMENTO À RETIRADA DO MERCADO
}

\author{
Drugs: from development to withdrawal
}

\author{
Filipe Galvão Ferreira ${ }^{1,2}$, Michelle Carneiro Polli, ${ }^{3,4}$, Yoko Oshima-Franco ${ }^{1} \mathrm{e}$ \\ Leonardo Fernandes Fraceto ${ }^{5,6^{*}}$
}

\author{
${ }^{1}$ Faculdade de Farmácia, Universidade de Sorocaba, Sorocaba, São Paulo, Brasil \\ ${ }^{2}$ Departamento de Farmacologia, Universidade Estadual Paulista Júlio de Mesquita Filho, Botucatu, São Paulo, \\ Brasil \\ ${ }^{3}$ Curso de Farmácia, Universidade São Francisco, Campinas, São Paulo, Brasil \\ ${ }^{4}$ Faculdade de Farmácia, PUC-CAMPINAS, Campinas, São Paulo, Brasil \\ ${ }^{5}$ Departamento de Engenharia Ambiental, Universidade Estadual Paulista Júlio de Mesquita Filho, Campus \\ Sorocaba, Av. Três de Março, 511, Alto da Boa Vista, CEP 18087-180, Sorocaba, São Paulo, Brasil \\ ${ }^{6}$ Departamento de Bioquímica, Instituto de Biologia, Unicamp, Campinas, São Paulo, Brasil
}

*Autor para correspondência: leonardo@sorocaba.unesp.br

Recebido em 17/01/2008 - Aceito em 11/01/2009

RESUMO: O desenvolvimento e a introdução de um novo fármaco na terapêutica são sempre acompanhados de expectativas quanto à sua utilização na prática clínica. Os períodos existentes desde a descoberta de um composto com atividade terapêutica até a sua introdução do mercado são conhecidos como fases pré-clínica e clínica. Estes ensaios destinam-se a responder a uma série de questionamentos científicos objetivando avaliar a segurança do novo composto bem como encontrar melhores opções terapêuticas. A utilização de medicamentos objetiva aumentar a expectativa de vida bem como erradicar doenças. Por outro lado, podem aumentar os custos da atenção à saúde se utilizados inadequadamente e/ou levar à ocorrência de reações adversas. Atualmente, são conhecidos os riscos inerentes ao uso de fármacos, o que levou as agências reguladoras a exigir estudos que comprovassem a segurança dos novos fármacos antes de permitir a sua comercialização. Este trabalho descreve casos de insucesso de fármacos clinicamente significativos como a talidomida, fenfluramina, dexfenfluramina, terfenadina, mibefradila, fenilpropanolamina, cerivastatina e rofecoxibe. Esses fármacos exemplificam casos de compostos que foram introduzidos na terapêutica, mas, por razões de toxicidade foram retirados mesmo após a realização de ensaios clínicos, evidenciando o papel fundamental da farmacovigilância nesse processo.

PALAVRAS-CHAVE: química farmacêutica, retirada de medicamentos do mercado, vigilância de produtos comercializados.

ABSTRACT: The development and introduction of a new drug in therapeutics are always followed by expectations about its utilization in clinical area. The periods between the finding of a compound with therapeutic activity and its introduction into the market are known as pre-clinical and clinical phases. These assays intend to answer to a series of scientific questions, aiming to evaluate the safety of the new compound as well as look for better therapeutic options. The use of pharmacological drugs aims to increase life expectation and eradicate diseases. On the other hand, such drugs might provoke a rise in the costs of health care if used inappropriately and/or provoke adverse reactions. Nowadays, the pharmacological drugs inherent risks are well known, what has taken the controlling agencies to demand studies able to prove new drugs safety before their authorization to be commercialized. This work describes some unsuccessful cases of clinical important drugs such as thalidomide, fenfluramine, dexfenfluramine, terfenadine, mibefradil, phenylpropanolamine, cerivastatine and rofecoxibe. These drugs exemplify cases of compounds introduced into therapeutics but, for toxicity reasons, removed even after clinical assays realization, evidentiating the essential role of pharmacovigilance in this process.

KEYWORDS: chemistry, pharmaceutical , drug withdrawal from market, product surveillance, postmarketing. 


\section{INTRODUÇÃO}

O desenvolvimento de um novo fármaco é dividido em várias etapas ou estágios que pode ser dividido em: (1) descoberta de um composto com atividade terapêutica; (2) estudos pré-clínicos e (3) estudos clínicos. Introduzir um novo fármaco na terapêutica é um processo longo, cerca de 12 anos em média e bastante oneroso, com probabilidade de sucesso pequena. De cada 100.000 novos compostos descobertos, apenas 250 são submetidos aos ensaios pré-clínicos e, apenas 5 entram em ensaios clínicos (testes em humanos). Ao final, estima-se que apenas uma molécula chegue ao mercado (LIMA et al., 2003), uma nova entidade química que seja patenteável e, para isto deve ser segura, eficaz e/ou melhore a qualidade de vida dos pacientes (HARDMAN, 2001).

Na década de 1950 o custo médio na pesquisa e desenvolvimento (P\&D) de um novo fármaco era de 1.500 .000 (hum milhão e meio de dólares) (Lima et al., 2003). Em seguida, ela precisa ser submetida a um rigoroso processo de desenvolvimento, que culminará na sua autorização para uso e comercialização (GRAHAME-SMITH e ARONSON, 2004).

Os estudos pré-clínicos têm o objetivo principal de avaliação farmacológica em sistemas in vitro e em animais in vivo para a obtenção do maior conhecimento possível acerca de suas propriedades e de seus efeitos adversos. Ao mesmo tempo, sua farmacocinética é testada em animais. Além disso, o composto é submetido a testes de toxicidade a curto e longo prazo em animais, para que suas propriedades farmacológicas possam ser definidas dentro de uma relação dose-resposta. A duração do teste toxicológico está relacionado com a provável duração do uso terapêutico. Neste estágio torna-se importante ainda a avaliação dos efeitos do composto sobre a fertilidade e a reprodução, testes de teratogenicidade e testes para mutagenicidade e carcinogenicidade. Também são realizados nesta fase testes relacionados com a estabilidade no novo composto, possibilidade de produção em larga escala bem como estudos de formulação (GRAHAME-SMITH e ARONSON, 2004).

Cerca da metade das substâncias falham durante a fase de desenvolvimento pré-clínico; para as restantes, um dossiê é preparado e submetido à autoridade reguladora (p. ex., FDA nos EUA e ANVISA, no Brasil), cuja permissão é necessária para proceder os estudos em seres humanos. Essa permissão não é obtida facilmente, e a autoridade reguladora pode negá-la ou exigir trabalho adicional a ser feito antes da aprovação (GRAHAME-SMITH e ARONSON, 2004).

Os ensaios clínicos são realizados em três fases: Fase I: representa a primeira administração do composto em humanos (30-100 voluntários) e deve seguir os preceitos éticos internacionais. Nesta fase utilizamse normalmente voluntários sadios e, eventualmente podem ser realizados em pacientes gravemente enfermos em circunstâncias nas quais a toxicidade elevada torna-se aceitável (pacientes com câncer, por exemplo). $O$ objetivo principal dessa fase é avaliar a tolerância em seres humanos e determinar a posologia segura (LIMA et al., 2003); Fase II: avalia-se a eficácia do medicamento em doentes (100-300), primeiramente em ensaios piloto e, posteriormente, em doentes selecionados, coletando-se, também, os dados de segurança do medicamento; Fase III - aumenta-se tanto a amostragem como diversifica-se a gama de pessoas nos testes. Nesta fase, também são incluídos tratamentos mais prolongados com o composto, cerca de 6 a 12 meses, visando à flexibilidade na dosagem, bem como a coleta de dados sobre segurança e eficácia do mesmo (PIMENTEL et al., 2006).

O método científico aplicado ao ensaio clínico necessita de um equilíbrio entre a aplicação da inferência indutiva (observar com base na teoria) com a inferência dedutiva (teorizar com base na observação). A prevalência da inferência dedutiva (preocupações com a teoria) levou a medicina a gerar inúmeras interpretações e evidências equivocadas. Citem-se como exemplos, dentre as mais recentes, as doses elevadas com que os beta-bloqueadores e os inibidores da enzima conversora de angiotensina (IECA) começaram a ser usados na terapêutica da hipertensão arterial, sem menção ao fracasso de muitos antiarrítmicos, como demonstrado mais recentemente (ECHT et al., 1992).

A fase IV, conhecida também como farmacovigilância é de responsabilidade do órgão regulamentador (PIMENTEL et al., 2006) e é o estudo do uso real do fármaco na prática médica. Esta vigilância, após a comercialização, que ocorre após a aprovação do FDA, é necessária para fornecer "feedback" do uso em grande escala do fármaco. Nesta fase, podem ser descobertos novos efeitos terapêuticos ou tóxicos, incluindo efeitos a longo prazo ou raros que não eram discerníveis em pequeno grupo de indivíduos (SEVALHO, 2001).

A importância da farmacovigilância é que em estudos clínicos de fase III, devido ao número de indivíduos estudados, efeitos raros podem não ser detectados, e em função do tempo de duração do ensaio, efeitos decorrentes do uso prolongado do medicamento podem não ser revelados. Torna-se importante destacar também que por força do próprio modelo experimental a que são submetidas às populações participantes da investigação, não devem ocorrer impropriedades nem por parte dos prescritores ao receitarem o medicamento, nem por parte dos usuários no cumprimento das prescrições. Além disso, em razão do controle experimental, não participam pacientes que possam apresentar riscos (patologias que não sejam alvo direto dos testes, uso concomitante de 
outros fármacos ou grupos específicos como gestantes, crianças ou idosos) para o estudo por apresentarem problemas clínicos. Assume-se, portanto, que os estudos de fase IV de farmacovigilância (vigilância póscomercialização) são sinônimos referentes ao mesmo processo de detecção, acompanhamento e controle de problemas decorrentes do uso já autorizado de medicamentos. Estes estudos são essenciais em relação aos medicamentos novos, pois proporcionam a avaliação de seu uso em grandes populações. A importância da farmacovigilância pode ser exemplificada pelos inúmeros fármacos que foram introduzidos na terapêutica e, posteriormente, por razões de toxicidade retirados (GRAHAME-SMITH e ARONSON, 2004).

O impacto do uso de medicamentos em uma sociedade tem várias facetas. Por um lado, os medicamentos podem aumentar a expectativa de vida, erradicar certas doenças, trazer benefícios sociais e econômicos; por outro lado, podem aumentar os custos da atenção à saúde se utilizados inadequadamente e/ou levar à ocorrência de reações adversas a medicamentos (PFAFFENBACH et al., 2002).

De acordo com a Organização Mundial de Saúde, reação adversa a medicamento (RAM) é definida como sendo qualquer evento nocivo e não intencional que ocorreu na vigência do uso de um medicamento, utilizado com finalidade terapêutica, profilática ou diagnóstica, em doses normalmente recomendadas (WORLD HEALTH ORGANIZATION, 1969).

O estudo sistemático de RAM ou farmacovigilância tem por objeto a detecção, avaliação, compreensão e prevenção dos riscos dos efeitos adversos dos medicamentos. Seus instrumentos de investigação podem ser clínicos, epidemiológicos, experimentais ou diagnósticos. Esses conhecimentos são importantes para que se possa usar um fármaco de modo racional, promovendo-se uma terapêutica mais adequada às necessidades dos pacientes e evitando-se riscos desnecessários. Muitos efeitos adversos a medicamentos são raros, e sua toxicidade não é previsível através de experimentos em animais ou de ensaios clínicos controlados. Então, o objetivo principal de um monitoramento de reações adversas a medicamentos é definir, o mais rápido possível, a capacidade de um medicamento produzir efeitos indesejáveis (PFAFFENBACH et al., 2002). Todos os medicamentos têm efeitos colaterais e, portanto, devem ser acompanhados após sua introdução no mercado (DAINESI, 2005).

Um entrave nessa questão é que, diferentemente do início do século $X X$, a velocidade com que um medicamento é difundido em nível mundial é muito maior, gerando sempre uma disputa entre o lado científico e o lado financeiro dos fabricantes de produtos farmacêuticos que buscam o lucro. A pesquisa acaba fornecendo resultados rápidos, e muitas vezes duvidosos, devido a essa pressão. Pouco se conhece da toxicologia do que é vendido atualmente, fato este devido ao alto custo desse estudo, do tempo relativamente longo, das muitas cobaias necessárias para realização de testes e da burocracia legal (PIMENTEL et al., 2006).

\section{AGÊNCIAS REGULADORAS}

A FDA (Food and Drug Administration) é a agência reguladora dos Estados Unidos da América que faz o controle dos alimentos (tanto humano como animal), suplementos alimentares, medicamentos (humano e animal), cosméticos, equipamentos médicos, materiais biológicos e produtos derivados do sangue humano. A Agência Européia de Medicamentos (EMEA) contribui para a proteção da saúde pública e animal, garantindo a segurança, eficácia e alta qualidade dos medicamentos para uso humano e veterinário. Ao reunir os recursos científicos dos 25 Estados-Membros numa rede de mais de 40 autoridades nacionais competentes, a EMEA coordena a avaliação e fiscalização de medicamentos em toda a União Européia (UE). A Agência trabalha em estreita cooperação com parceiros internacionais, reforçando a contribuição da UE para a harmonização global.

No Brasil, foi criada a Agência Nacional de Vigilância Sanitária pela Lei nำ.782, de 26 de janeiro de 1999. A finalidade institucional da Agência é promover a proteção da saúde da população por intermédio do controle sanitário da produção e da comercialização de produtos e serviços submetidos à vigilância sanitária, inclusive dos ambientes, dos processos, dos insumos e das tecnologias a eles relacionados.

Essas agências são responsáveis pelo monitoramento dos medicamentos presentes no mercado, sendo a FDA a referência. Elas possuem a autoridade de introduzir e/ou retirar um medicamento do mercado, buscando qualidade, eficácia e segurança.

\section{CASOS DE INSUCESSO DE FÁRMACOS}

A retirada de fármacos do mercado tem ocorrido desde 1937, nos EUA, por conseqüência do uso de um xarope de sulfanilamida contendo como veículo o dietilenoglicol, que causou a morte de 100 pessoas. O número de fármacos retirados pela FDA tem aumentado nas últimas décadas por razões de segurança (Tabela 1) (GENERALI, 2001). Alguns fármacos foram substituídos ou indicados para uso restrito. Aproximadamente 22 fármacos foram retirados do mercado dos Estados Unidos desde 1982, sendo que $77 \%$ na ultima década. Novas reações adversas severas são imediatamente transmitidas pela mídia, e as autoridades regulatórias são forçadas a tomar medidas drásticas proporcionando sua retirada do mercado. Os casos publicados de rabdomiólise associados com o uso de cerivastatina (Baycol® - Bayer) representam um exemplo clássico. 
Embora os efeitos causados pelo fármaco já haviam sido descritos como um possível efeito adverso da cerivastatina, muitos médicos falharam reconhecendo apenas tardiamente os sintomas relatados por seus pacientes. Embora a responsabilidade do fabricante nos eventos trágicos fosse admitida, ao mesmo tempo se torna limitada; e a validação do fármaco, quando usado corretamente, nunca foi avaliada.

O alarme do público em relação às reações adversas publicados pela mídia vem apresentando numerosos protestos contra as indústrias farmacêuticas, uma vez que, em muitos casos, isso é muito pouco documentado ou limita as prescrições. Além disso, se alguma reação ocorrer isoladamente é possível convencer os fabricantes a retirar espontaneamente o fármaco do mercado (PREZIOSI, 2004).

Entre setembro de 1997 a setembro de 1998, 5 medicamentos citados a seguir foram retirados do mercado por motivo de reações adversas inesperadas. Essas ações levantaram inúmeras questões sobre a segurança dos fármacos que são introduzidos no mercado (WITHDRAWALS OF FDA, 1998; THE FDA AND DRUG SAFETY, 1998; FRIEDMAN et al., 1999).

\section{FÁRMACOS RETIRADOS DO MERCADO}

\section{Talidomida}

A talidomida foi sintetizada primeiramente como sedativo, em 1953, e causou um dos mais dramáticos episódios da história da medicina. Em 1957, foi comercializada como fármaco sedativo-hipnótico, amplamente vendido em países europeus, asiáticos, no Canadá e América do Sul, tornando-se o medicamento mais vendido na Alemanha Ocidental para o tratamento da insônia (POWELL, 1996; RAJE, 1999). A despeito de sua ampla comercialização nos continentes Europeu e Asiático, este fármaco, em diferentes especialidades farmacêuticas: Kavadon $^{\circledR}$, Sedalis ${ }^{\circledR}$, Softenon ${ }^{\circledR}$, Distaral ${ }^{\circledR}$, Contergan ${ }^{\circledR}$, entre outros, não obteve licença da FDA dos Estados Unidos da América para uso neste país (LIMA et al., 2001).

No ínicio da década de 60, a talidomida foi prescrita mundialmente como fármaco sedativo e antiemético, indicada no alívio do mal-estar matinal comum em gestantes (DALLY, 1998), sendo responsável pelo nascimento de milhares de crianças com deformações congênitas (focomelia), que foram, posteriormente atribuídas ao seu perfil teratogênico (LIMA et al., 2001).

A constatação do perfil teratogênico da talidomida, representou um marco na conscientização do risco da administração de um fármaco em sua forma racêmica quando a razão eudísmica entre os dois enantiômeros (R,S) é desconhecida (LIMA et al., 2001). No caso da talidomida, o enantiômero $R$ é ativo e o $S$ é responsável pelo efeito teratogênico. Entretanto, torna-se inviável administrar o enantiômero $R$ (puro) responsável pela atividade terapêutica, uma vez que este é metabolizado e convertido ao enantiômero S provocando o efeito teratogênico.

Assim, após três anos de comercialização e após intensa pressão da imprensa, a talidomida foi retirada do mercado, em 1961, deixando para trás o trágico saldo de 8.000 crianças com malformação congênita em 46 países, inclusive no Brasil. Nos Estados Unidos o medicamento nunca foi liberado, pois a FDA achou que deveria aprofundar a experimentação devido a ocorrências de neurite periférica e alterações tireoidianas (BORGES e FRÖEHLICH, 2003).

Quatro anos mais tarde, em 1965, o médico israelita Jacob Sheskin prescreveu a talidomida como sedativo para pacientes leprosos, observando fortuitamente acentuada redução da dor e do processo inflamatório associado ao leproma, identificando as propriedades antiinflamatórias, até então desconhecidas. Este intrigante perfil antiinflamatório incentivou diversos grupos de pesquisas ao estudo do provável mecanismo de ação relacionado às ações benéficas da talidomida sobre os lepromas. Em 1991, foi demonstrado, em trabalho pioneiro, que pacientes leprosos apresentavam níveis sanguíneos aumentados de um modulador do sistema imunológico, identificado como uma citocina denominada fator de necrose tumoral a (TNFa) (KAPLAN et al., 1991). Estes pesquisadores constataram, posteriormente, a participação da talidomida na inibição seletiva desta citocina, quando expressa em quantidade superior àquela necessária à manutenção do sistema imunológico normal, denotando, portanto, o potencial imunorregulador (LIMA et al., 2001).

Com base na descoberta das propriedades antiinflamatórias e imunorreguladoras da talidomida, a indústria norte-americana Celgene Co. solicitou à FDA sua aprovação para uso no tratamento da hanseníase, concedida em julho de 1998. Marcava-se de forma definitiva o renascimento deste fármaco, que representa, atualmente, um dos principais agentes terapêuticos disponíveis para o tratamento efetivo dos lepromas (CALDERON et al., 1997).

Trabalhos paralelos divulgados por vários laboratórios de pesquisa, demonstram a aplicação terapêutica potencial da talidomida no câncer, interferindo com o processo de angiogênese patológica, na AIDS, mais especificamente no retardamento da replicação do HIV, e no auxílio da perda de peso desordenada de portadores do vírus da imunodeficiência adquirida e do bacilo da tuberculose (LIMA et al., 2001). Adicionalmente, a talidomida representa a primeira terapia disponível nos últimos 20 anos para o tratamento de mieloma múltiplo (RAJE, 1999).

\section{Fenfluramina e Dexfenfluramina}


Em setembro de 1997, foram retirados do mercado 2 fármacos indicados como supressores do apetite, a fenfluramina (Pondimin®) e a dexfenfluramina (Redux®) respectivamente, após apresentar relatos de doença valvular cardíaca (FRIEDMAN et al., 1999). Dois meses antes foram investigados 24 casos de doença valvular cardíaca caracterizada especificamente pela patologia das válvulas e pela regurgitação da aorta e da mitral em pacientes que faziam o uso da associação fenfluramina + fertermina (CONNOLLY et al., 1997; MARK et al., 1997). A FDA publicou o problema, além de estudos epidemiológicos em pacientes que usavam fenfluramina e a dexfenfluramina ou em associação com a fertermina (FRIEDMAN et al., 1999).

A fenfluramina foi aprovada pela FDA em 1993, mas seu uso não se estendeu até o final da década de 90. Esse fármaco começou a ser utilizado em associação com uma outra substância inibidora do apetite, a fertermina. Essa combinação tornou-se bastante popular para perda de peso. No entanto, a FDA não havia aprovado a utilização da fenfluramina em associação, nem o seu uso para um período prolongado. Anteriormente sabia-se sobre problemas relacionados às válvulas cardíacas, contudo, a FDA aprovou a dexfenfluramina para uso isolado por mais de 1 ano.

Inicialmente, os estudos mostraram que aproximadamente $30 \%$ dos pacientes que faziam uso da associação (fenfluramina + fertermina) tinham regurgitação da aorta e/ou da mitral onde somente $1 \%$ da população exibiu essa anormalidade (KHAN et al., 1998; JICK et al., 1998; DEVEREUX, 1998). Nem a FDA, nem os fabricantes e nem a comunidade médica esperavam que a doença das valvas seria o resultado tóxico da fenfluramina ou dexfenfluramina. Depois desse estudo entre o uso dessa associação e a doença da valva, concluiu-se que esses fármacos deveriam ser retirados do mercado (FRIEDMAN et al., 1999).

\section{Terfenadina}

O anti-histamínico terfenadina, cuja utilização terapêutica havia sido aprovada em 1985, é na realidade um pró-fármaco, e após sua absorção, é rapidamente convertido no metabólito ativo fexofenadina, apresentandose como um fármaco seguro quando usado isoladamente. Entretanto, 0 aumento da concentração da terfenadina (que pode ocorrer quando sua metabolização está inibida) no organismo foi associado com arritmia cardíaca potencialmente fatal. Os fármacos inibidores da enzima citocromo P450, como por exemplo, eritromicina ou cetoconazol podem ser os responsáveis pelo aparecimento de arritmias quando utilizados em associação com a terfenadina (HONIG et al., 1992; HONIG et al., 1993).

Essa interação não era conhecida no momento da sua aprovação. Depois de reconhecer essa interação, a FDA desenvolveu uma crítica investigação laboratorial para explicar e demonstrar a interação conclusiva. Concomitantemente, os médicos reduziram significativamente a sua prescrição (FRIEDMAN et al., 1999). O fabricante da terfenadina, Hoechst Marion Roussel desenvolveu um novo medicamento (Allegra ${ }^{\circledR}$ ) contendo como princípio ativo a fexofenadina, o metabólito ativo da terfenadina. A fexofenadina fornece os mesmos efeitos da terapia da terfenadina e pode ser administrado seguramente com outros medicamentos comumente prescritos (BERNSTEIN et al., 1997; MARKHAM e WAGSTAFF, 1998; BRONSKY et al., 1998). A FDA propôs, então, a substituição da terfenadina pela fexofenadina, pois sua terapia é idêntica e não apresenta riscos à saúde dos pacientes (FRIEDMAN et al., 1999).

\section{Mibefradila}

Em junho de 1998, dois fármacos foram retirados do mercado após o fabricante e a FDA descobrirem a magnitude e a gravidade dos efeitos adversos não detectados nos ensaios pré-clínico e clínico. Mibefradila, um bloqueador do canal de cálcio, foi aprovado para o tratamento de pacientes com hipertensão e angina crônica estável. Sua retirada ocorreu após observações de interações prejudiciais, em potencial, com outros medicamentos (ROCHE CITES DRUG INTERACTIONS IN MIBEFRADIL WITHDRAWAL, 1998).

A mibefradila administrada isoladamente não causa nenhum efeito tóxico (Friedman et al., 1999). Esse fármaco reduz significativamente a atividade das enzimas do citocromo P450, principalmente a isoenzima CYP3A4 (KRAHENBUHL et al., 1998; MULLINS et al., 1998; SCHMASSMANN-SUHIJAR et al., 1998). Essas enzimas, localizadas principalmente no fígado, são essenciais para a eliminação de fármacos presentes no organismo. Na presença da mibefradila, quando associado com outro fármaco, este se bioacumula chegando a níveis tóxicos.

A FDA publicou uma nota em dezembro de 1997 relatando que pacientes que faziam uso da mibefradila apresentaram efeitos adversos quando administrado com outros medicamentos. Publicou também, uma carta divulgando o problema aos profissionais da saúde (FRIEDMAN et al., 1999). Com a farmacovigilância e a contínua aparição de efeitos adversos, a FDA reconheceu que a mibefradila era amplamente utilizada e tornouse comum a interação com outros fármacos; analisando seu risco/benefício, decretou sua retirada do mercado. Atualmente, sabe-se que a mibefradila interage com 26 tipos de fármacos diferentes, sendo inviável sua permanência no mercado (FRIEDMAN et al., 1999).

\section{Fenilpropanolamina}


A fenilpropanolamina é uma amina simpatomimética sintética, que foi utilizada como descongestionante em produtos para tosse, resfriado e em supressores do apetite, os quais requerem ou não prescrição médica. Esta amina esteve no mercado farmacêutico, por muitos anos, mas preocupações com o uso seguro da mesma foram levantadas, desde 1969, através da publicação de casos nos quais o uso de fenilpropanolamina isolada ou em combinação com outros fármacos foi associado ao desenvolvimento de hemorragia cerebral, particularmente, após a administração, em mulheres jovens, da primeira dose de agentes supressores do apetite contendo fenilpropalamina (HUMBERSTONE, 1969; ARAÚJO, 2000).

Recentemente, resultados de um estudo de tipo caso-controle envolvendo homens e mulheres de 18 a 49 anos de idade, os quais foram hospitalizados em decorrência de hemorragia subaracnóide ou hemorragia intracerebral, indicou que mulheres que administram supressores de apetite contendo fenilpropanolamina aumentam, em 15 vezes, quando comparadas com outras mulheres, a chance de sofrer hemorragia cerebral, enquanto que aquelas que utilizam, pela primeira vez, medicamentos para tosse ou resfriados contendo fenilpropanolamina, aumentam em três vezes a chance de sofrer esta complicação (ARAÚJO, 2000).

O estudo desenvolvido pelo grupo da Universidade de Yale contou com 702 pacientes, entre os quais 425 (61\%) apresentaram hemorragia subaracnóide e 277 (39\%) apresentaram hemorragia cerebral. Como controle foram utilizados 1376 indivíduos (ARAÚJO, 2000). Embora o risco de se desenvolver hemorragia cerebral, através do uso de fenilpropanolamina ou de produtos contendo fenilpropanolamina atualmente disponíveis no mercado seja muito pequeno, a seriedade de uma hemorragia cerebral e a incapacidade de se prever quais os indivíduos sob o risco de desenvolver tal complicação levaram a FDA a tomar medidas contra o seu uso. A comissão da FDA concluiu que os riscos associados ao uso de medicamentos contendo fenilpropanolamina ultrapassavam os benefícios que tais produtos possam trazer, principalmente, porque os mesmos são utilizados para tratar condições não graves.

Em 6 de novembro de 2000, a FDA advertiu os profissionais da saúde, a indústria farmacêutica e a população americana quanto aos riscos do uso de fenilpropanolamina e anunciou que tomaria as devidas providências para remover a fenilpropanolamina da composição de todos os medicamentos disponíveis no mercado. Solicitou ainda, que a indústria farmacêutica retirasse voluntariamente do mercado os produtos contendo fenilpropanolamina. A FDA alertou consumidores sobre os riscos desse fármaco, solicitando à população para evitar o uso de fenilpropanolamina ou buscando a utilização de medicamentos alternativos que não contivessem essa substância na formulação, presente em cerca de 400 produtos (ARAÚJO, 2000). Em resposta à FDA, indústrias farmacêuticas, como a SmithKline Beecham, Bayer Corporation e Novartis, anunciaram o compromisso de atender a solicitação do governo de retirar os medicamentos e de manter a confiança que consumidores sempre tiveram em seus produtos.

\section{Cerivastatina}

Os inibidores da hidroximetilglutaril-coenzima-A (HMG-CoA) redutase, também conhecidos como estatinas, são os fármacos mais potentes e eficazes para reduzir o LDL colesterol. Desde que foram aprovados para uso no tratamento da hipercolesterolemia pela FDA, em 1987, diversos estudos clínicos vêm demonstrando que esses medicamentos são capazes de reduzir eventos cardiovasculares, quer na prevenção primária quer na prevenção secundária da doença arterial coronariana (SHEPHERD et al., 1995).

A HMG-CoA redutase é uma enzima que catalisa a conversão da HMG-CoA a ácido mevalônico, precursor do colesterol. Esta enzima é alvo para a intervenção farmacológica porque ela atua na biossíntese do colesterol. Em nível celular, portanto, as estatinas inibem a conversão da HMG-CoA para mevalonato e como conseqüência, a síntese celular do colesterol (CORSINI et al., 1999). De modo geral, são bem tolerados, porém alguns pacientes podem desenvolver toxicidade hepática e/ou muscular, em graus variados. Com relação aos efeitos tóxicos sobre a musculatura esquelética, a complicação mais séria observada com o uso dessas medicações é a rabdomiólise (PASTERNAK et al., 2002), em que pese a dificuldade em se conceituar adequadamente a rabdomiólise (MAGALHÃES, 2005).

A FDA avaliou 3.339 casos de rabdomiólise relacionada ao uso de estatina entre janeiro de 1990 e março de 2002. A cerivastatina foi a estatina que mais apresentou problemas (THOMPSON et al., 2003). O mecanismo exato pelo qual as estatinas podem causar a rabdomiólise não se encontra ainda completamente esclarecido. Diversas hipóteses são possíveis: depleção de metabólitos intermediários da síntese do colesterol (reduzindo os níveis de ácido mevalônico), induzem a apoptose celular e podem causar alterações nos canais de condutância ao cloro dentro dos miócitos (MAGALHÃES, 2005).

Observações recentes com a cerivastatina demonstraram que a administração de mevalonato foi capaz de melhorar os efeitos tóxicos causados pela cerivastatina, sugerindo que as ações deletérias deste fármaco podiam ser devidas à redução desses compostos intermediários, particularmente o ácido mevalônico, mais do que a ação tóxica direta da mesma (GEMICl et al., 2001).

Outra possível explicação para a toxicidade muscular das estatinas se relaciona com os canais de cloro intracelular. Essas estruturas, no músculo, são responsáveis pela hiperpolarização da célula e como conseqüência, do seu relaxamento. Modificações na permeabilidade da membrana celular também podem resultar do bloqueio desse sistema (IGEL et al., 2001). Têm sido descritas alterações nas propriedades da membrana celular com a utilização desses medicamentos. Algumas estatinas, mais lipofílicas, podem atravessar mais 
facilmente a membrana celular em função dessas alterações e, por conseguinte, resultar em maior potencial para o desenvolvimento de agressão muscular (MAGALHÃES, 2005).

Além disso, muitos casos de rabdomiólise ocorreram concomitantemente com outras doenças (como por exemplo, a insuficiência renal) ou sendo associado com outro fármaco (por exemplo, gemfibrozil, o qual foi responsável por 31 das 52 mortes registradas nos Estados Unidos). Em outros casos, a cerivastatina era usada na dose de 0,8 mg por dia, aprovada pela FDA para uso clínico, mesmo sendo uma dose mais elevada daquela recomendada pelo fabricante para iniciar o tratamento.

\section{Rofecoxibe}

Na década de 1960, pesquisas mostraram que os salicilatos eram muito bons para o alivio da dor na artrite reumatóide, mas com os esteróides, seu uso ficou limitado devido sua toxicidade (JÜNI e DIEPPE, 2004). As indústrias de maior porte começaram a desenvolver os antiinflamatórios não esteroidais (AINES) chegando a vender mais de 20 bilhões de dólares por ano.

Na década de 1980, o benoxaprofeno (Opren®) era comercializado com base em um único mecanismo de ação, que claramente foi associado aos seus efeitos adversos, incluindo fotossensibilidade e hepatotoxicidade (JÜNI et al., 2002). O fabricante manteve a comercialização do fármaco até que foi obrigado a retirar do mercado quando pacientes idosos foram a óbito por insuficiência hepática após o seu uso (TAGGART e ALDERDICE, 1982).

Há pouco mais de dez anos, duas isoformas da cicloxigenase - COX-1 e COX-2 - foram descobertas, com diferentes padrões de expressão. As propriedades antiinflamatórias dos AINEs foram relacionadas à inibição de COX-2, enquanto os efeitos adversos gastrintestinais ocorriam devido à inibição de COX-1 (KARGMAN et al., 1996). A partir daí, enfatizou-se a toxicidade gastrintestinal dos AINES, ignorando-se outros eventos adversos (DIEPPE et al., 2004).

Um dos primeiros AINES a ser muito comercializado como inibidor seletivo da COX-2, eficaz e não tendo problema de toxicidade foi o Celecoxibe (Celebrex®). O agente subseqüente foi o rofecoxibe (Vioxx®), produzido pela Merck Sharp \& Dohme. Em 2000, um grupo de estudo denominado VIGOR (BOMBARDIER et al., 2000) (comparou $50 \mathrm{mg}$ de rofecoxibe a $500 \mathrm{mg}$ de naproxeno) mostrou uma redução dos efeitos gastrintestinais com os novos antiinflamatórios em seis meses de uso. Nesse estudo, ocorreram mais eventos adversos graves e trombóticos com rofecoxibe em comparação ao naproxeno. A incidência de infarto do miocárdio foi menor nos pacientes do grupo naproxeno do que nos que receberam o rofecoxibe. A explicação do produtor sobre esses resultados foi de que o risco cardiovascular não se associava a rofecoxibe, mas sim que o naproxeno conferia proteção cardiovascular. Agora, porém, se sabe que naproxeno, na dose de $500 \mathrm{mg}$, duas vezes ao dia, também acarreta risco cardiovascular, e a FDA adverte que a dose máxima é de $200 \mathrm{mg}$, duas vezes ao dia (WANNMACHER, 2005).

Em 2001, o volume de vendas de rofecoxibe atingiu US $\$ 2,5$ bilhões no mercado de 80 países, graças à vigorosa campanha de marketing (WANNMACHER, 2005). Em 2004, dizia-se que o volume mundial de vendas atingiu dez bilhões de dólares. Em 2003, Fitz Gerald publicou que celecoxibe e rofecoxibe suprimiam a formação de prostaglandina $I_{2}$ dependente de COX-2 em voluntários sadios. $A \mathrm{PGI}_{2}$ inibe a agregação plaquetária, causa vasodilatação e previne a proliferação de células musculares lisas endoteliais. Contrariamente, os fármacos não afetavam tromboxana $A_{2}$, que tem efeitos opostos. Isso poderia predispor pacientes a infarto do miocárdio e acidente vascular encefálico trombótico (WANNMACHER, 2005).

Em 30 setembro de 2004 a Merck Sharp \& Dohme-Chibret anunciou a retirada de rofecoxibe do mercado mundial, em razão de risco relativamente maior de infarto de miocárdio e acidentes vasculares cerebrais, confirmados após 18 meses do início do tratamento em pacientes que receberam VIOXX ${ }^{\circledR}$ (15 por 1000 pacientes/ano) em comparação com aqueles do grupo placebo (7,5 por 1000 pacientes/ano) no ensaio clínico multicêntrico, randomizado e duplo-cego APPROVe (Adenomatous Polyp Prevention on VIOXX), iniciado em 2000 e projetado para durar três anos, avaliando a eficácia do rofecoxibe $(25 \mathrm{mg})$ na prevenção da recorrência de pólipos colorretais em pacientes com histórico de adenomas. O estudo incluiu 2.600 pacientes e, em 2004, foi precocemente suspenso. Apenas duas semanas antes, o medicamento havia sido liberado para crianças com artrite reumatóide (WANNMACHER, 2005).

No período entre sua liberação e a retirada do comércio (cinco anos), dois milhões de pacientes usaram rofecoxibe. Os investigadores da FDA estimaram ter havido quase 28 mil infartos do miocárdio e mortes súbitas nos Estados Unidos, entre 1999 e 2003 (WANNMACHER, 2005). Este episódio e alguns outros temas posteriores levantaram muitas questões importantes relacionadas à política de aprovação de medicamentos, às evidências cientificas e ao papel da farmacovigilância, sendo uma desconfortável conseqüência, a erosão da confiança pública, difícil de ser recuperada (DAINESI, 2005).

\section{CONCLUSÕES}

A farmacovigilância tem um papel fundamental nesse processo, ao centralizar informações de possíveis reações adversas e monitorar os fármacos disponíveis no mercado. Os estudos clínicos necessitam aperfeiçoar 
seus procedimentos para obter maior rigor em relação aos novos fármacos e evitar problemas mais sérios com a introdução de novos fármacos, de modo a garantir a qualidade e a segurança dos medicamentos.

Paradoxalmente, quase 50 anos após o incidente teratogênico da década de 60 , a talidomida ressurge como fármaco promissor para o tratamento da hanseníase, AIDS e doenças crônicas degenerativas (inclusive no Brasil, em programas oficiais). Entretanto, a despeito das novas aplicações terapêuticas desse fármaco, seu emprego requer rigorosa avaliação da relação risco-benefício, exigindo, em alguns casos, o monitoramento médico.

\section{REFERÊNCIAS BIBLIOGRÁFICAS}

ARAÚJO, R.C. Os riscos do uso da fenilpropanolamina: As bases científicas para a retirada do mercado. Pharmacia Brasileira. v.6, p. 71-73, 2000.

BERNSTEIN, D.I.; SCHOENWETTER, W.F.; NATHAN, R.A. et al. Efficacy and safety of fexofenadine hydrochloride for treatment of seasonal allergic rhinitis. Ann Allergy Asthma Immunol. v.79, p.443-448, 1997.

BOMBARDIER, C.; LAINE, L.; REICIN, A.; SHAPIRO, D.; BURGOS-VARGAS, R.; DAVIS, B. et al. Comparison of upper gastrointestinal toxicity of rofecoxib and naproxen in patients with rheumatoid arthritis. VIGOR Study Group. New England Journal of Medice. v.343, p.1520-1528, 2000.

BORGES, L.G.; FRÖEHLICH, P.E. Thalidomide: new perspectives for its use as antiinflammatory, immunossupressive and antiangiogenic drug. Revista da Associação Médica Brasileira. v.49, n.1, p.96-102, 2003.

BRONSKY, E.A.; FALLIERS, C.J.; KAISER, H.B.; AHLBRANDT, R.; MASON, J.M.; Effectiveness and safety of fexofenadine, a new nonsedating $\mathrm{H} 1$-receptor antagonist in the treatment of fall allergies. Allergy Asthma Proc. v.19, p.135-141, 1998.

CALDERON, P.; ANZILOTTI, M.; PHELPS, R. Thalidomide in dermatology. New indications for an old drug. International Journal of Dermatology. 1997;36(12): 881-7, 1997.

CONNOLLY, H.M.; CRARY, J.L.; MCGOON, M.D. et al. Valvular heart disease associated with fenfluraminephertermine. New England Journal of Medicine. v.337, p.581-588, 1997.

CORSINI A, BELLOSTA S, BAETTA R. New insight sinto the pharmacodynamic and pharmacokinetic properties of statins. Pharmacology \& Therapeutics. v.84, p.413-428, 1999.

DAINESI, S.M. Pharmacovigilance: More than ever, an overall responsibility. Clinics. v.60, n.4, p.267-270, 2005.

DALLY, A. Thalidomide: was the tragedy preventable? Lancet. v.351, p.1197-1199, 1998.

DEVEREUX, R.B. Appetite-supressants and valvular heart disease. New England Journal of Medicine. v.339, p.765-766, 1998.

DIEPPE, P.; BARTLETT, C.; DAVEY, P.; DOYAL, L.; EBRAHIM, S. Balancing benefits and harms: the example of non-steroidal anti-inflammatory drugs. British Medical Journal. v.329, p.31-34, 2004.

DIEPPE, P.A.; EBRAHIM, S.; MARTIN, R.M.; JÜNI, P. Lessons from the withdrawal of rofecoxib. British Medical Journal. v.329, p.867-868, 2004.

ECHT, D.S.; LIEBSON, P.R.; MITCHELL, L.B. et al. Mortality and morbidity in patients receiving eucainide, flucainide or placebo. The Cardiac Arrhythmia Suppression Trial. New England Journal of Medicine. v.324, p.781788, 1992.

FRIEDMAN, M.A.; WOODCOCK, J.; LUMPKIN, M.M.; SHUREN, J.E.; HASS, A.E.; THOMPOSON, L.J. The safety of newly approved medicines. Journal of American Medical Association. v.281, p.1728-1734, 1999.

GEMICI, G.; TOPRAK, A.; OATKAY, A. Rhabdomyolysis due to cerivastatin monotherapy. American Journal of Medicine. v.110, p.742, 2001.

GENERALI, J.A. Drug withdrawals in the US: 1980 to 2001, Drug facts and comparison news 43-45 (Facts and Comparisons, a Wolters Kluwer Company, St. Louis, 2001).

GRAHAME-SMITH, D.G.; ARONSON, J.K. Tratado de farmacologia clínica e farmacoterapia. $3^{\text {rd }}$.ed. Rio de Janeiro. p.83, 2004. 
HARDMAN, J.G.; LIMBIRD, L.E.; GILMAN, A.G. Goodman \& Gilman's. The Pharmacological basis of therapeutics. Québec: McGraw-Hill, 2001.

HONIG, P.K.; WOOSLEY, R.L.; ZAMANI, K.; CONNER, D.P.; CANTILENA, L.R.J. Changes in the pharmacokinetics and electrocardiographic pharmacodynamics of terfenadine with concomitant administration of erythromycin. Clinical Pharmacology \& Therapeutics. v.52, p.231-238, 1992.

HONIG, P.K.; WOOSLEY, R.L.; ZAMANI, K,.; CONNER, D.P.; MULLIN, J.C.; CANTILENA, L.R. TerfenadineKetoconazole interaction. Journal of American Medical Association. v.269, p.1513-1518, 1993.

HUMBERSTONE PM. Hypertension and cold remedies. British Medical Journal. I:846, 1969.

IGEL, M.; SUDHOP, T.; VON BERGMANN, K. Metabolism and drug interation of 3-hydroxy-3-methylglutaryl coenzime A-reductase inhibitors (statins). European Journal of Clinical Pharmacology. v.57, p.357-364, 2001.

JICK, H.; VASILAKIS, C.; WEINRAUCH, L.A.; MEIER, C.R.; JICK, S.S.; DERBY, L.E. A population-based study of appetite-supressant drugs and the risk of cardiac-valve regurgitation. New England Journal of Medicine. v.339, p.719-724, 1998.

JÜNI, P.; DIEPPE, P. Older people should NOT be prescribed "coxibs" in place of conventional NSAIDs. Age Ageing. v.33, p.100-104, 2004.

JÜNI, P.; RUTJES, A.W.; DIEPPE, P.A. Are selective COX 2 inhibitors superior to traditional non steroidal antiinflammatory drugs? British Medical Journal. v.324, p.1287-1288, 2002.

KAPLAN, G.; SAMPAIO, E.P.; SARNO, E.N.; GALILY, R.; COHN, Z.A. Thalidomide selectively inhibits tumor necrosis factor alpha production by stimulated human monocytes. Journal of Experimental Medicine. v.173, n.3, p.699-703, 1991.

KARGMAN, S.; CHARLESON, S.; CARTWRIGHT, M.; FRANK, J.; RIENDEAU, D.; MANCINI, J. et al. Characterization of Prostaglandin $\mathrm{G} / \mathrm{H}$ Synthase 1 and 2 in rat, dog, monkey, and human gastrointestinal tracts. Gastroenterology. v.111, p.445-454, 1996.

KHAN, M.A.;, HERZOG, C.A.; ST PETER, J.V. et al. The prevalence of cardiac valvular insufficiency assessed by transthoracic echocardiography in obese patients treated with appetite-supressant drugs. New England Journal of Medicine. v.339, p.713-718, 1998.

KRAHENBUHL, S.; MENAFOGLIO, A.; GIOSTRA, E.; GALLINO, A. Serious interaction between mibefradil and tacrolimus. Transplantation. v.66, p.1113-1115, 1998.

LIMA, J.S.; REZA, D.; TEIXEIRA, S.; COSTA, C. Pesquisa Clínica: fundamentos, aspectos éticos e perspectivas. Revista da Socerj. v.16, n.4, p.225-233, 2003.

LIMA, L.M.; FRAGA, C.A.M.; BARREIRO, E.J. O renascimento de um fármaco: talidomida. Química Nova. v.24, n.5, p.683-688, 2001.

MAGALHÃES, M.E.C. Mecanismos de rabdomiólise com as estatinas. Arquivos brasileiros de cardiologia. v.85, p.42-44, 2005.

MARK, E.J.; PATALAS, E.D.; CHANG, H.T.; EVANS, R.J.; KESSIER. S.C. Fatal pulmonary hypertension associated with short-term use of fenfluramine and phertermine. New England Journal of Medicine. v.337, p.602606, 1997.

MARKHAM, A.; WAGSTAFF, A.J. In discussion of: fexofenadine. Drugs. v.55, p.269-276, 1998.

MULLINS, M.E.; HOROWITZ, B.Z.; LINDEN, D.H.; SMITH, G.W.; NORTON, R.L.; STUMP, J. Life-threatening interaction of mibefradil and $\beta$-blockers with dihydropyridine calcium channel blockers. Journal of American Medical Association. v.280, p.157-158, 1998.

PASTERNAK, R.C.; SMITH, S.C.; BAIREY-MERZ, C.N. ACC/AHA/NHLBI clinical advisory on the use and safety of statins. Journal of the American College of Cardiology. v.40, p.567-572, 2002. 
PFAFFENBACH, G.; CARVALHO, O.M.; BERGSTEN-MENDES, G. Reações adversas a medicamentos como determinantes da admissão hospitalar. Revista da Associação Médica Brasileira. v.48, n.3, p.237-241, 2002.

PIMENTEL, L.C.F.; CHAVES, C.R.; FREIRE, L.A.A.; AFONSO, J.C. O inacreditável emprego de produtos químicos perigosos no passado. Química Nova. v.29, n.5, p.1138-1149, 2006.

POWELL, R.J. New roles for thalidomide. British Medical Journal. v.313, p.377, 1996.

PREZIOSI, P. Science, pharmacoeconomics and ethics in drug R\&D: a sustainable future scenario? Nature. v.3, p.521-526, 2004.

RAJE, N.; ANDERSON, K. Thalidomide--a revival story. New England Journal of Medicine. v.341, n.21, p.1606$1609,1999$.

ROCHE CITES DRUG INTERACTIONS IN MIBEFRADIL WITHDRAWAL. American Journal of Health-System Pharmacy. v.55, p.1445, 1998.

SCHMASSMANN-SUHIJAR, D.; BULLINGHAM, R.; GASSER, R. et al. Rhabdomyolysis due to interaction of simvastatin with mibefradil. Lancet. v.351, p.1929-1930, 1998.

SEVALHO, G. Farmacovigilância: Bases Históricas, conceituais e operacionais. In: Gomes MJVM, Reis AMM. Ciências Farmacêuticas: Uma abordagem em farmácia hospitalar. $1^{\text {st }}$.ed. São Paulo, p.109, 2001.

SHEPHERD, J.; COBBE, S.M.; FORD, I. Prevention of coronary heart disease with pravastatin in men with hypercholesterolemia. New England Journal of Medicine. v.333, p.1301-1307, 1995.

TAGGART, H.M.; ALDERDICE, J.M. Fatal cholestatic jaundice in elderly patients taking benoxaprofen. British Medical Journal (Clin Res Ed) v.284, p.1372, 1982.

THE FDA AND DRUG SAFETY. Delaware Medical Journal. v.70, p.405-406, 1998.

THOMPSON, P.D.; CLARKSON, P.; KARAS, R.H. Statin-Associated Myopathy. Journal of American Medical Association. v.289, p.1681-1690, 2003.

WANNMACHER, L. Inibidores seletivos de cicloxigenase-2 revisitados um ano depois. Uso racional de medicamentos. v.2, n.2, p. 1-6 2005.

WITHDRAWALS OF FDA - APPROVED DRUGS RAISE QUESTIONS. Mayo Clinic Health Letter. v.16, p.4, 1998.

WORLD HEALTH ORGANIZATION. International drug monitoring: the role of the hospital. Report of a WHO meeting. Geneva: WHO; p.1-24, 1969. 
Tabela 1: Fármacos retirados do mercado por razões de segurança (1982-2002).

\section{Seção 1.01 Fármacos Seção 1.02 Classe terapêutica Seção 1.03 Principal problema detectado}

\begin{tabular}{|c|c|c|}
\hline Benoxapreno & AINE & Icterícia fatal \\
\hline Nomifensina & Antidepressivo & Anemia hemolítica \\
\hline Suprofeno & AINE & Síndrome da dor de flanco \\
\hline Ticrinafeno & Diurético & Hepatotóxico \\
\hline Zomepiraco & AINE & Reações anafiláticas \\
\hline Cianidanol & Hepatoprotetores e Lipotrópicos & Anemia hemolítica \\
\hline Indoprofeno & AINE & Hepatotóxico \\
\hline Isoxicam & AINE & Necrose da epiderme \\
\hline Suloctidila & Antitrombótico & Hepatotóxico \\
\hline Zimelidina & $\begin{array}{l}\text { Antidepressivo (Inibidor Seletivo } \\
\text { da Recaptação de Serotonina) }\end{array}$ & Reações de hipersensibilidade com envolvimento neurológico \\
\hline Alosetron & $\begin{array}{l}\text { Antagonista do receptor de } \\
\text { serotonina }\end{array}$ & Colite isquêmica, constipação severa \\
\hline Astemizol & Anti-histamínico & Arritmia cardíaca \\
\hline Bronfenaco & AINE & Hepatotóxico \\
\hline Cerivastatina & $\begin{array}{l}\text { hipocolesterolêmico ou anti- } \\
\text { lipêmico }\end{array}$ & Rabdomiólise \\
\hline Cisaprida & $\begin{array}{l}\text { Parassimpatomimético que age } \\
\text { como agonista de serotonina }\end{array}$ & Eventos cardiovasculares \\
\hline Dexfenfluramina & Supressor do apetite & Regurgitação da aorta e da mitral \\
\hline Encainida & Anti-arritmico & $\begin{array}{l}\text { Elevada taxa de morte em pacientes com arritmias assintomática } \\
\text { seguida de recente ataque do coração }\end{array}$ \\
\hline Fenfluramina & Supressor do apetite & Regurgitação da aorta e da mitral \\
\hline Fenilpropanolamina & Descongestionante & Hemorragia cerebral \\
\hline Flosequinana & Vasodilatador & $\begin{array}{l}\text { Os efeitos benéficos dos sintomas da insuficiência cardíaca não } \\
\text { duraram além dos três primeiros meses de terapia }\end{array}$ \\
\hline Grepafloxacino & Antibacteriano & Eventos cardiovasculares \\
\hline Levometadil & Opióide & Eventos cardíacos severos \\
\hline Mibefradila & Bloqueador do canal de cálcio & $\begin{array}{l}\text { Inibição das enzimas do Citocromo P450, resultando em } \\
\text { diversas interações significantes }\end{array}$ \\
\hline Rapacuronío & $\begin{array}{l}\text { Relaxante muscular do grupo } \\
\text { aminoesteróide }\end{array}$ & Broncoespasmo e fatalidades inexplicadas \\
\hline Terfenadina & Anti-histamínico & Arritmias cardíacas \\
\hline Temafloxacino & Antibiótico & $\begin{array}{l}\text { Hipoglicemia em idosos, assim como anemia hemolítica, } \\
\text { frequentemente associada com insuficiência renal, testes com } \\
\text { marcadores hepáticos alterados e problemas de coagulação }\end{array}$ \\
\hline Troglitazona & Antidiabético & Hepatotóxico \\
\hline
\end{tabular}

